\title{
Cognitive Biases Affecting the Maintenance of COVID-19 Pandemic
}

\author{
Kawthar Mohamed ${ }^{1}$, Niloufar Yazdanpanah ${ }^{1}$, Amene Saghazadeh ${ }^{1}$, and Nima Rezaei ${ }^{1}$ \\ ${ }^{1}$ Affiliation not available
}

August 28, 2020

\begin{abstract}
All the countries and regions have already been infected with novel coronavirus disease (COVID-19). This super small guest has paralyzed the economy of the entire world, from the extreme fall of the oil prices to the bankruptcy of the great companies or even the small retail shops. The people's lifestyle is undergoing significant changes, by which it is leaving a negative impact on their psychological and physical health. The atmosphere is filled with dual accusations from each one of the governments and their citizens. Recognizing cognitive biases that have potentially affected decision-making during the COVID-19 pandemic would help in considering some behavioral changes for curbing this global viral infection.
\end{abstract}

\section{Cognitive Biases Affecting the Maintenance of COVID-19 Pandemic}

Kawthar Mohamed ${ }^{1,2}$, Niloufar Yazdanpanah ${ }^{1,2}$, Amene Saghazadeh ${ }^{2,3}$, and Nima Rezaei ${ }^{2,4,5}$

1. MetaCognition Interest Group (MCIG), Universal Scientific Education and Research Network (USERN), Tehran, Iran

2. Research Center for Immunodeficiencies, Children's Medical Center, Tehran University of Medical Sciences, Tehran, Iran

3. Systematic Review and Meta-analysis Expert Group (SRMEG), Universal Scientific Education and Research Network (USERN), Tehran, Iran

4. Department of Immunology, School of Medicine, Tehran University of Medical Sciences, Tehran, Iran

5. Network of Immunity in Infection, Malignancy and Autoimmunity (NIIMA), Universal Scientific Education and Research Network (USERN), Tehran, Iran

Corresponding author: Prof. Nima RezaeiMailing address: Children's Medical Center Hospital, Dr. Qarib St, Keshavarz Blvd, Tehran 14194, IranTel: +9821-6692-9234; Fax: +9821-6692-9235Email:rezaei_nima@tums.ac.ir, rezaei_nima@yahoo.comRunning title: COVID-19 Cognitive biases

\section{Abstract}

All the countries and regions have already been infected with novel coronavirus disease (COVID-19). This super small guest has paralyzed the economy of the entire world, from the extreme fall of the oil prices to the bankruptcy of the great companies or even the small retail shops. The people's lifestyle is undergoing significant changes, by which it is leaving a negative impact on their psychological and physical health. The atmosphere is filled with dual accusations from each one of the governments and their citizens. Recognizing cognitive biases that have potentially affected decision-making during the COVID-19 pandemic would help in considering some behavioral changes for curbing this global viral infection.

Keywords: COVID-19; decision-making; media bias; management; meta-cognitive bias; statistical bias 


\section{Introduction}

Novel coronavirus disease (COVID-19), an outbreak that developed to a pandemic ${ }^{1}$, is a complex literal problem that accompanies a lot of intricacies and solutions. In the resultant chaotic situation, the brain, in order to counteract the high weight of uncertainty, would assess its prior knowledge, connect it to the current complex problem, and apply the best solution. The tendency to the same thing is called cognitive bias. Cognitive biases are vividly present in decisions taken in critical conditions, and the COVID-19 crisis is not an exception ${ }^{2}$.

\section{Cognitive biases and decision-making at the government level}

COVID-19 is a prototype of scenarios that accurately portrays a sequential emergence of different cognitive biases and related defects in decision-making that at least in part have played a role in the widespread of the disease. Initially, it was thought that COVID-19 resembles the severe acute respiratory syndrome (SARS) pandemic in merely involving the eastern part of Asia, and it is not spreading through other regions. This is called anchoring bias, which means to judge based on the first received information without considering the undergoing alterations through the time period. Subsequently, leaders started to believe this small enemy can attack each and every country in the world. However, this time, the Western countries from Italy to the US had the idea of not being much exposed to this pandemic, and they ignored repetitive warning facts, plus being confident about their strong infrastructural ability to cope with the pandemic. These two ideas are called confirmation bias and overconfidence effect, respectively. Next, the choice supportive bias came on the scene, being represented in the UK choice of the herd-immunity strategy as the primary approach to overcome the pandemic and their ignorance of all other facts that can potentially defeat the efficiency of this strategy $y^{3,4}$.

Other cognitive biases involved in the context of declining people's trust of the governments during the COVID-19 pandemic include the Dunning-Kruger effect, selective perception bias, and optimism bias. The Dunning-Kruger effect occurs when a person overestimates his knowledge about a certain topic, despite the limited available data in that area. The idea of injecting disinfectants to patients with COVID-19 and the consideration of COVID-19 as a cold or flu are a few examples of this bias committed by some heads of government.

The idea of thinking of COVID-19 as flu might also implicate the selective perception bias, by which messages and actions are usually perceived according to the one's frame of reference, and any other contradictive messages and facts are not considered at all. Finally, attempts of some governments to promote the use of unapproved drugs as miracles in the treatment of COVID-19 resulted in a bias that is called Ostrich bias or Optimism bias ${ }^{4,5}$.

The mentioned cognitive biases are only those which could be detected in the literature. There are, of course, other cognitive biases that remained elusive due to the lack of public announcements, particularly in countries other than the US and European countries.

\section{Statistical biases and decision-making at the healthcare system level}

Statistical biases have affected medical decision-making during the COVID-19 pandemic. Accordingly, some issues need to be considered when reporting statistics of COVID-19 at the country level and the state level. First, given the unequal distribution of population within the country, the use of aggregated data from country-level to calculate the state or national statistics might result in the biased overall estimated COVID-19 growth rate due to the higher spread rate and number of death in hotspot regions ${ }^{6}$. Second, the population of each country must be considered when the number of infected patients is reported. As the population of a country increases, the number of infected cases is expected to potentially increase, and this 
is an issue needed to be considered in determining the severity of countries' states ${ }^{7}$. Third, the number of performed COVID-19 tests is a crucial factor to be considered when comparing the condition of countries in terms of the number of confirmed cases. As the number of performed cases in a country increases, the number of diagnosed asymptomatic cases increases, and this results in a high disease rate report for that country. Indeed, this country is prosperous in detecting a large percentage of both symptomatic and asymptomatic cases and isolates them to cease the infection spread, while the reported rate can add biases that the mentioned country has failed in disease control.

\section{Media biases and decision-making at the social level}

It is a common trend for using social and news media to track disease outbreaks. In particular, at the beginning of an outbreak, people have a dominant tendency to pick up the information from informal media rather than official sources since the data released by the official sources might be delayed by a few weeks. When official sources start covering outbreak news, the primary interest of people might shift to the information coming from official sources, while continuing to use informal media as alternative sources of data. Overall, informal media, as well as official sources complementarily, contribute to the community's understanding of the epidemiology of an emerging outbreak.

During the COVID-19 outbreak, there has been an increasing interest in publishing posts on social media. By using the data available in these social environments, the exponential growth rate of COVID-10 could be estimated to fall within the range of 1.42 to $2.64^{8}$. The rate is comparable to that reported by published articles for the same duration ${ }^{9}$, implying the reliability of data gathered from social media. However, unreliable posts published in social media are an issue of rising concern, because, whereas the number of unreliable posts is less than that of reliable posts published in the social media, the number of reactions to unreliable posts is much more than that to reliable posts. During the COVID-19 outbreak, Twitter has appeared as a neutral social media ${ }^{10}$, YouTube, and Reddit as the media of cutting unreliable data and Gab as the one of amplifying unreliable posts ${ }^{8}$.

The engagement in social media was most pronounced on January 20, 2020, when the world health organization (WHO) declared the novel coronavirus as a pandemic species - spreading as quickly as more than four million cases being affected in only four months. Consequently, the COVID-19 became the subject of an infodemic - referred to as the circulation of misinformation about the disease ${ }^{8}$. Effects of this infodemic which is mostly formed by informal media - on the people's perception have been extended to the behavior and action of people leading to a further worsening of the COVID-19 outbreak. To exemplify, CNN, on March 8, 2020, announced that Italy would impose a lockdown in the northern region. Therefore, a substantial portion of the northern population decided to travel to the southern region. It caused overcrowding in trains and airports, and since then, Italy increasingly encountered the new cases of COVID-19 for two weeks.

Now unreliable posts have the power to affect people's perception disproportionally; it is necessary to avoid the sharing of these posts in the first place. Studies show that the diffusion of unreliable posts concerning the COVID-19 outbreak is largely due to non-thinking, and the force should be primarily directed to those who share the posts. Analytical thinking is an effective intervention for the problem. In the study ${ }^{11}$, when people simply received a reminder to analyze the issue and judge the accuracy of the claim, they were more likely to indeed discriminate between true and false content on COVID-19.

\section{Conclusions}

The emergence of the COVID-19 outbreak was not a choice, but its persistence might be a result of the cognitive biases that primarily affect medical, strategic, and consumer decision-making. Due to the lack of sufficient knowledge, there are many scientific uncertainties associated with the COVID-19 pandemic, and 
uncertainties are a potential source of meta-cognitive bias and, therefore, poor performance. Such cognitive biases broadly consist of the following four categories: prior hypotheses and focusing on limited targets, exposure to limited alternatives, insensitivity to outcome probabilities, and the illusion of manageability 12. For a strategic decision-making process, cognitive biases happen at the level of research, education, and execution. A systematic review of studies identified 19 cognitive biases that threaten medical decision-making

13. They can occur at the screening, diagnostic, and therapeutic levels. Biases imposed by unreliable data or delayed data release occur at different data sharing levels. Figure 1 is a schematic illustration of the levels of decision-making under the influence of different cognitive biases. In this manner, a rational mode of action should not be only limited to decision-making in a single area of interest but it requires decision makers in different areas of management to frame the least biased outlet of decision-making.

\section{Declarations}

Ethics approval and consent to participate. Not applicable

Consent for publication. Not applicable

Availability of data and materials. Not applicable

Competing interests. The authors declare that they have no competing interests

Funding. There is no funding for the present study

Acknowledgments. Not applicable

Figure legends

Figure 1. Potential cognitive biases affecting the maintenance of COVID-19 pandemic.

\section{References}

1. Hanaei S, Rezaei N. COVID-19: Developing from an Outbreak to A Pandemic. Archives of Medical Research. 2020.

2. Dong Y, Mo X, Hu Y, et al. Epidemiological characteristics of 2143 pediatric patients with 2019 coronavirus disease in China.Pediatrics. 2020.

3. Marques CF. Why Our Leaders Fail to Learn Pandemic Lessons

2020; https://www.bloombergquint.com/gadfly/coronavirus-biases-are-playing-role-in-poor-pandemicleadership. Accessed 13th May 2020, 2020.

4. Pohl RF. Cognitive Illusions

Intriguing Phenomena in Judgement, Thinking and Memory.Psychology Press; 2016.

5. Tisdall S. From Trump to Erdoğan, men who behave badly make the worst leaders in a pandemic. 2020; https://www.theguardian.com/commentisfree/2020/apr/26/trump-to-erdogan-men-whobehave-badly-make-worst-leaders-pandemic-covid-19. Accessed 13th May 2020, 2020.

6. Burghardt K, Lerman K. Systematic Biases in Aggregated COVID-19 Growth Rates. arXiv preprint arXiv:200412994. 2020.

7. De Mesnard L. Tracking COVID-19 pandemic: The per-capita approach changes the whole picture. 2020.

8. Cinelli M, Quattrociocchi W, Galeazzi A, et al. The covid-19 social media infodemic. arXiv preprint arXiv:200305004. 2020.

9. Liu Y, Gayle AA, Wilder-Smith A, Rocklöv J. The reproductive number of COVID-19 is higher compared

to SARS coronavirus. Journal of Travel Medicine. 2020;27(2). 
10. Yang K-C, Torres-Lugo C, Menczer F. Prevalence of Low-Credibility Information on Twitter During the COVID-19 Outbreak. arXiv preprint arXiv:200414484. 2020.

11. Pennycook G, McPhetres J, Zhang Y, Rand D. Fighting COVID-19 misinformation on social media: Experimental evidence for a scalable accuracy nudge intervention. 2020.

12. Das TK, Teng BS. Cognitive biases and strategic decision processes: An integrative perspective. Journal of management studies.1999;36(6):757-778.

13. Blumenthal-Barby JS, Krieger H. Cognitive biases and heuristics in medical decision making: a critical review using a systematic search strategy. Medical Decision Making. 2015;35(4):539-557.

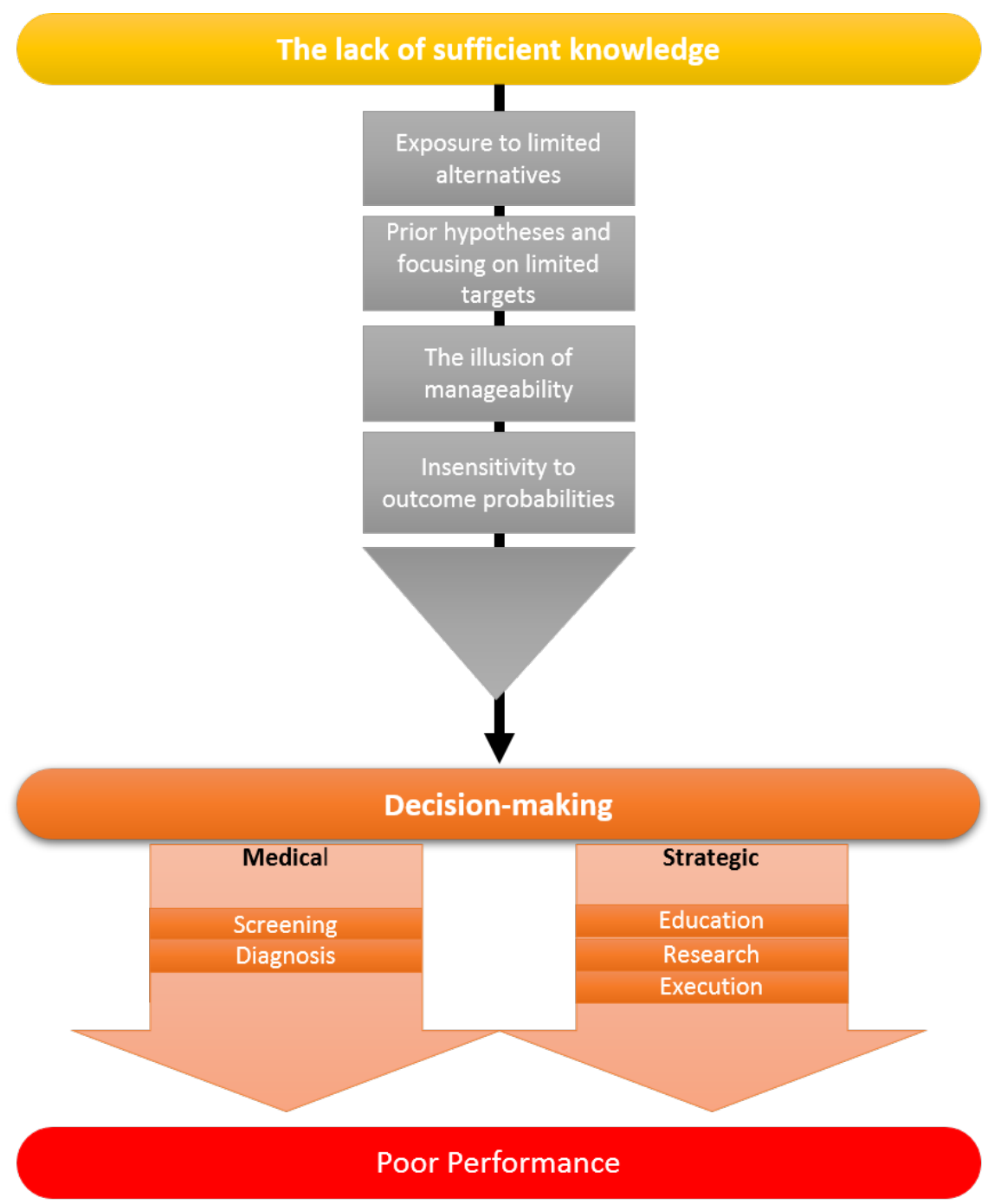

Figure 1. Potential cognitive biases affecting the maintenance of COVID-19 pandemic. 


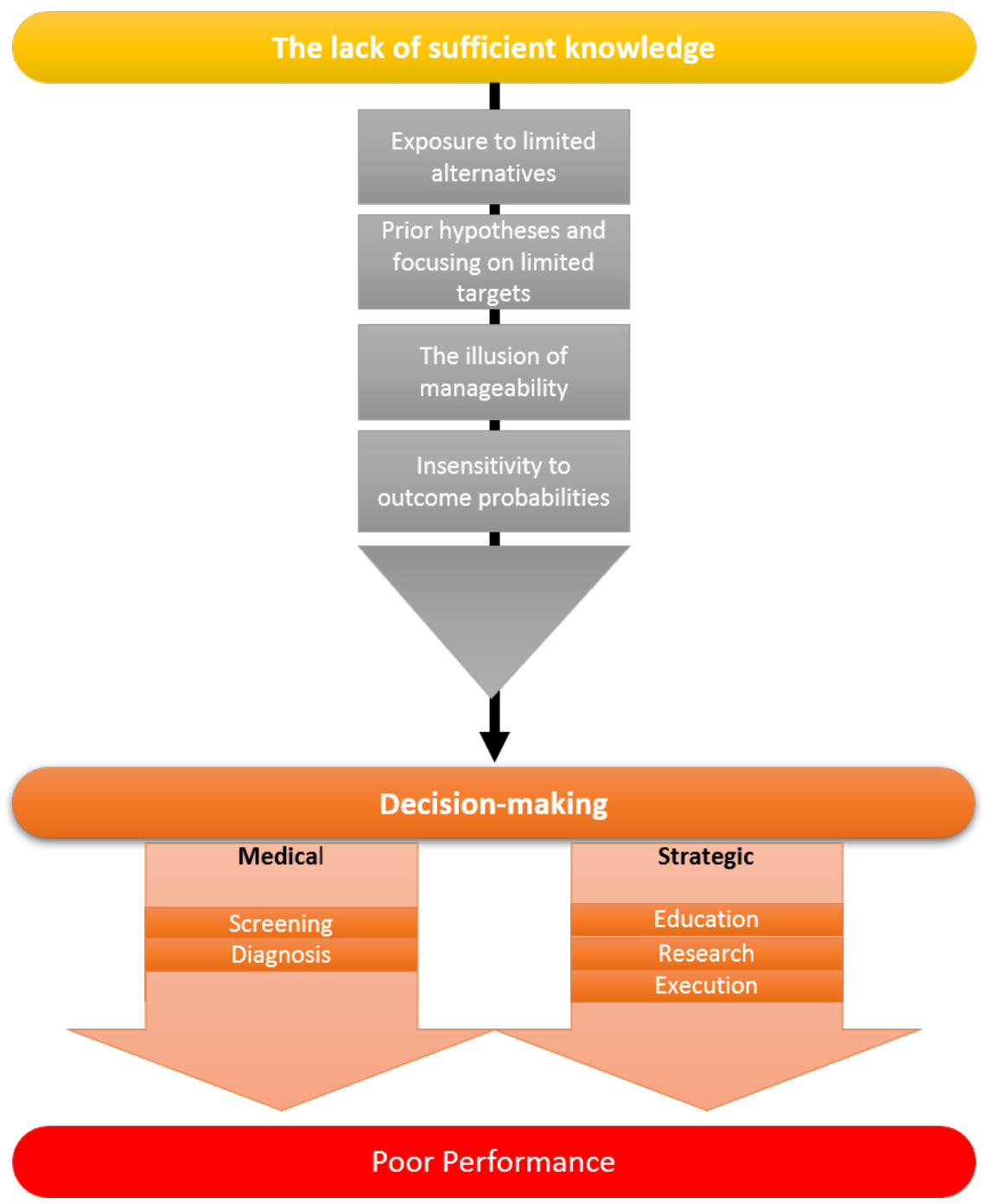

